\title{
ON ODD PERFECT NUMBERS. III
}

\section{SURYANARAYANA}

It is not known whether or not odd perfect numbers exist. However, many interesting necessary conditions for an odd integer to be perfect have been found out. A bibliography of previous work on odd perfect numbers is given by McCarthy [3].

Throughout this paper $n$ denotes an odd perfect number. The following results have been proved in [4], [6] and [5] respectively:

(i) $\prod_{p / n} p /(p-1)<(175 / 96) \zeta(3)<2.19125$.

(ii) $n$ is of the form $12 t+1$ or $36 t+9$.

(iii) If $n$ is of the form $36 t+9$ and $5 \mid n$, then

$$
\sum_{p \mid n} \frac{1}{p}<\frac{1}{3}+\frac{1}{5}+\frac{1}{13}+\log \left(\frac{65}{61}\right) \quad(\sim 0.674) .
$$

(iv) If $n$ is of the form $36 t+9$ and $5 \nmid n$, then

$$
\sum_{p \mid n} \frac{1}{p}<\frac{1}{3}+\frac{1}{338}+\log \left(\frac{18}{13}\right) \quad(\sim 0.662) .
$$

The object of this paper is to improve the upper bound for the product $\prod_{p / n} p /(p-1)$ given by (i) above. We prove the following:

THEOREM. ( $\alpha$ ) If $n$ is of the form $12 t+1$ and $5 \mid n$,

$$
2<\prod_{p \mid n} \frac{p}{p-1}<\frac{56791}{33612} \cdot \zeta(3)<2.031002 .
$$

( $\beta$ ) If $n$ is of the form $12 t+1$ and $5 \nmid n$,

$$
2<\prod_{p \mid n} \frac{p}{p-1}<\frac{1760521}{1050375} \cdot \zeta(3)<2.014754 .
$$

( $\gamma$ ) If $n$ is of the form $36 t+9$ and $5 \mid n$,

$$
2<\prod_{p \mid n} \frac{p}{p-1}<\frac{318897}{177023} \cdot \zeta(3)<2.165439 .
$$

(ס) If $n$ is of the form $36 t+9$ and $5 \nmid n$,

$$
2<\prod_{p \mid n} \frac{p}{p-1}<\frac{3706148208}{2125240975} \cdot \zeta(3)<2.096234 .
$$

Received by the editors July 14, 1966. 
Proof. Euler proved that $n$ must be of the form $p_{0}^{\alpha_{0}} \cdot x^{2}$, where $p_{0}$ is a prime of the form $4 \lambda+1, \alpha_{0}$ is of the form $4 \mu+1, x>1$ and $\left(p_{0}, x\right)$ $=1$. Hence we can write $n=p_{0}^{\alpha_{0}} p_{1}^{\alpha_{1}} p_{2}^{\alpha_{2}} \cdots p_{k}^{\alpha_{l}}$, where $\alpha_{r}$ is even for $1 \leqq r \leqq k$. We shall suppose without loss of generality that $p_{1}<p_{2}$ $<\cdots<p_{k}$. Let $\sigma(n)$ denote the sum of all the positive divisors of $n$. Since $n$ is a perfect number, we have $\sigma(n)=2 n$, from which it can easily be seen that

$$
\begin{aligned}
\prod_{r=0}^{k} \frac{p_{r}}{p_{r}-1} & =2 \prod_{r=0}^{k}\left(1-p_{r}^{-\left(\alpha_{r}+1\right)}\right)^{-1} \\
& >2 .
\end{aligned}
$$

Throughout the following $q_{r}$ denotes the $r$ th prime, counting 2 as the first prime. We make use of the following well-known identity due to Euler:

$$
\prod_{r=1}^{\infty}\left(1-q_{r}^{-3}\right)^{-1}=\zeta(3),
$$

where $\zeta(s)$ is the Riemann Zeta function.

(a) Suppose $n$ is of the form $12 t+1$. In this case, it has been proved in $[4, p .134]$ that $p_{0}$ is of the form $12 N+1$ and hence $p_{0} \geqq 13$.

$\left(\mathrm{a}_{1}\right)$ Suppose $5 \mid n$ and $7 \mid n$. Then $p_{1}=5, p_{2}=7$. Now $\alpha_{2} \geqq 4$. For, if $\alpha_{2}=2$, then $\sigma\left(p_{2}^{\alpha_{2}}\right)=3.19$ and since $\sigma(n)=2 n$, it would follow that $3 \mid n$, which can not hold.

$\left(a_{1.1}\right)$ If $p_{0}=13$, then $p_{3} \geqq 11$ and $p_{r} \geqq q_{r+3}$ for $4 \leqq r \leqq k$. Since $\alpha_{r}$ is even for $1 \leqq r \leqq k, \alpha_{2} \geqq 4$ and $\alpha_{0} \geqq 1$, we have

$$
\begin{aligned}
\prod_{r=0}^{k}\left(1-p_{r}^{-\left(\alpha_{r}+1\right)}\right)^{-1}< & \left(1-13^{-2}\right)^{\cdots-1}\left(1-5^{-3}\right)^{-1}\left(1-7^{-5}\right)^{-1}\left(1-11^{-3}\right)^{-1} \\
& \times \prod_{r=4}^{k}\left(1-p_{r}^{-3}\right)^{-1} \\
< & \left(1-13^{-2}\right)^{-1}\left(1-5^{-3}\right)^{-1}\left(1-7^{-5}\right)^{-1}\left(1-11^{-3}\right)^{--1} \\
& \times \prod_{r=7}^{\infty}\left(1-q_{r}^{-3}\right)^{-1} \\
= & \frac{\left(1-2^{-3}\right)\left(1-3^{-3}\right)\left(1-7^{-3}\right)\left(1-13^{-3}\right)}{\left(1-1^{-2}\right)\left(1-7^{-5}\right)} \cdot \zeta(3) \\
= & \frac{56791}{67224} \cdot \zeta(3) .
\end{aligned}
$$


$\left(a_{1.2}\right)$ If $p_{0} \neq 13$, then since $p_{0}$ is of the form $12 N+1, p_{0} \geqq 37$. $p_{r} \geqq q_{r+2}$ for $3 \leqq r \leqq k$. Hence

$$
\begin{aligned}
\prod_{r=0}^{k}\left(1-p_{r}^{-\left(\alpha_{r}+1\right)}\right)^{-1} & <\left(1-37^{-2}\right)^{-1}\left(1-5^{-3}\right)^{-1}\left(1-7^{-5}\right)^{-1} \prod_{r=5}^{\infty}\left(1-q_{r}^{-3}\right)^{-1} \\
& <\frac{56791}{67224} \cdot \zeta(3) .
\end{aligned}
$$

Hence, by $(A),(\alpha)$ follows in the case $\left(a_{1}\right)$.

$\left(\mathrm{a}_{2}\right)$ Suppose $5 \mid n$ and $7 \nmid n$, then $p_{1}=5$ and $p_{r} \geqq q_{r+3}$ for $2 \leqq r \leqq k$. Since $\alpha_{0}$ is odd, $\left(1+p_{0}\right) \mid \sigma\left(p_{0}^{\alpha_{0}}\right)$ and hence $\left\{\left(1+p_{0}\right) / 2\right\} \mid n$, since $\sigma(n)=2 n$. Now, $p_{0} \neq 13$. For, otherwise, by the above, it would follow that $7 \mid n$, which is not the case. Since $p_{0}$ is of the form $12 N+1, p_{0} \geqq 37$. Hence

$$
\begin{aligned}
\prod_{r=0}^{k}\left(1-p_{r}^{-\left(\alpha_{r}+1\right)}\right)^{-1} & <\left(1-37^{-2}\right)^{-1}\left(1-5^{-3}\right)^{-1} \prod_{r=5}^{\infty}\left(1-q_{r}^{-3}\right)^{-1} \\
& <\frac{56791}{67224} \cdot \zeta(3) .
\end{aligned}
$$

Hence, by $(\mathrm{A}),(\alpha)$ follows in this case also. Thus $(\alpha)$ is proved.

$\left(\mathrm{a}_{3}\right)$ Suppose $5 \nmid n$ and $7 \mid n$, then $p_{1}=7$. Now, $\alpha_{1} \geqq 4$. For, if $\alpha_{1}=2$, it would follow as in $\left(\mathrm{a}_{1}\right)$ that $3 \mid n$, which does not hold.

$\left(\mathrm{a}_{3.1}\right)$ If $p_{0}=13$, then $p_{2} \geqq 11$ and $p_{r} \geqq q_{r+4}$ for $3 \leqq r \leqq k$. Hence

$$
\begin{aligned}
\prod_{r=0}^{k}\left(1-p_{r}^{-\left(\alpha_{r}+1\right)}\right)^{-1} & <\left(1-13^{-2}\right)^{-1}\left(1-7^{-5}\right)^{-1}\left(1-11^{-3}\right)^{-1} \prod_{r=7}^{\infty}\left(1-q_{r}^{-3}\right)^{-1} \\
& =\frac{\left(1-2^{-3}\right)\left(1-3^{-3}\right)\left(1-5^{-3}\right)\left(1-7^{-3}\right)\left(1-13^{-3}\right)}{\left(1-13^{-2}\right)\left(1-7^{-5}\right)} \cdot \zeta(3) \\
& =\frac{1760521}{2100750} \cdot \zeta(3) .
\end{aligned}
$$

$\left(a_{3.2}\right)$ If $p_{0} \neq 13$, then $p_{0} \geqq 37$ and $p_{r} \geqq q_{r+3}$ for $2 \leqq r \leqq k$. Hence

$$
\begin{aligned}
\prod_{r=0}^{k}\left(1-p_{r}^{-\left(\alpha_{r}+1\right)}\right)^{-1} & <\left(1-37^{-2}\right)^{-1}\left(1-7^{-5}\right)^{-1} \prod_{r=5}^{\infty}\left(1-q_{r}^{-3}\right)^{-1} \\
& <\frac{1760521}{2100750} \cdot \zeta(3) .
\end{aligned}
$$

Hence, by $(A),(\beta)$ follows in case $\left(a_{3}\right)$. 
$\left(\mathrm{a}_{4}\right)$ If $5 \nmid n$ and $7 \nmid n$, then $p_{r} \geqq q_{r+4}$ for $1 \leqq r \leqq k$. As in $\left(\mathrm{a}_{2}\right), p_{0} \neq 13$ and hence $p_{0} \geqq 37$. Hence

$$
\begin{aligned}
\prod_{r=0}^{k}\left(1-p_{r}^{-\left(\alpha_{r}+1\right)}\right)^{-1} & <\left(1-37^{-2}\right)^{-1} \prod_{r=5}^{\infty}\left(1-q_{r}^{-3}\right)^{-1} \\
& <\frac{1760521}{2100750} \cdot \zeta(3) .
\end{aligned}
$$

Hence, by (A), $(\beta)$ follows in this case also. Thus $(\beta)$ is proved.

(b) Suppose $n$ is of the form $36 t+9$. Since $3 \mid n, p_{1}=3$.

$\left(b_{1}\right)$ If $5 \mid n$, then $7 \nmid n$ in virtue of the result that $3 \cdot 5 \cdot 7$ does not divide $n$ (proved by Kuhnel, p. 203 of [2]).

(b1.1) Suppose $p_{0}=5$.

$\left(b_{1.1 .1}\right)$ If $11 \mid n$, then $\alpha_{0}=1$ in virtue of the result that $3 \cdot 5^{2} \cdot 11$ does not divide $n$ (proved by Kanold [1, p. 26]). In this case $p_{2}=11$ and $p_{r} \geqq q_{r+3}$ for $3 \leqq r \leqq k$. Further, $\alpha_{2} \geqq 4$. For, if $\alpha_{2}=2$, then $\sigma\left(p_{2}^{\alpha_{2}}\right)=133$ $=7.19$ and since $\sigma(n)=2 n$, it would follow that $7 \mid n$, which is not the case. Also, $\alpha_{1} \geqq 4$. For, if $\alpha_{1}=2$, then $\sigma\left(p_{1}^{\alpha_{1}}\right)=\sigma\left(3^{2}\right)=13 \mid n$ and this implies that

$$
\sum_{p \mid n} \frac{1}{p}>\frac{1}{3}+\frac{1}{5}+\frac{1}{11}+\frac{1}{13}>\frac{1}{3}+\frac{1}{5}+\frac{1}{13}+\log \left(\frac{65}{61}\right),
$$

a contradiction to (iii). Hence

$$
\begin{aligned}
\prod_{r=0}^{k}\left(1-p_{r}^{-(\alpha+1)}\right)^{-1} & <\left(1-5^{-2}\right)^{-1}\left(1-3^{-5}\right)^{-1}\left(1-11^{-5}\right)^{-1} \prod_{r=6}^{\infty}\left(1-q_{r}^{-3}\right)^{-1} \\
& <\frac{318897}{354046} \cdot \zeta(3) .
\end{aligned}
$$

Hence, by (A), $(\gamma)$ follows in this case.

$\left(b_{1.1 .2}\right)$ Suppose $11 \nmid n$. Then $\alpha_{1} \neq 4$. For, if $\alpha_{1}=4$, then $\sigma\left(p_{1}^{\alpha_{1}}\right)$ $=121=11^{2}$ and since $\sigma(n)=2 n$, it would follow that $11 \mid n$, which is not the case.

Hence, either $\alpha_{1}=2$ or $\alpha_{1} \geqq 6$.

Suppose $\alpha_{1}=2$. Then $\sigma\left(p_{1}^{\alpha_{1}}\right)=13 \mid n$ and in this case both 17 and 19 together do not divide $n$. For, otherwise, it would follow that

$$
\sum_{p ! n} \frac{1}{p}>\frac{1}{3}+\frac{1}{5}+\frac{1}{13}+\frac{1}{17}+\frac{1}{19}>\frac{1}{3}+\frac{1}{5}+\frac{1}{13}+\log \left(\frac{65}{61}\right),
$$

a contradiction to (iii). Hence 


$$
\begin{gathered}
\prod_{r=0}^{k}\left(1-p_{r}^{-\left(\alpha_{r}+1\right)}\right)^{-1}<\left(1-5^{-2}\right)^{-1}\left(1-3^{-3}\right)^{-1}\left(1-13^{-3}\right)^{-1}\left(1-17^{-3}\right)^{-1} \\
\times \prod_{r=9}^{\infty}\left(1-q_{r}^{-3}\right)^{-1}
\end{gathered}
$$

if $17 \mid n, 19 \nmid n$; and

$$
\begin{gathered}
\prod_{r=0}^{k}\left(1-p_{r}^{-\left(\alpha_{r}+1\right)}\right)^{-1}<\left(1-5^{-2}\right)^{-1}\left(1-3^{-8}\right)^{-1}\left(1-13^{-3}\right)^{-1}\left(1-19^{-3}\right)^{-1} \\
\times \prod_{r=9}^{\infty}\left(1-q_{r}^{-3}\right)^{-1}
\end{gathered}
$$

if $17 \nmid n, 19 \mid n$; and

$$
\prod_{r=0}^{k}\left(1-p_{r}^{-\left(\alpha_{r}+1\right)}\right)^{-1}<\left(1-5^{-2}\right)^{-1}\left(1-3^{-3}\right)^{-1}\left(1-13^{-3}\right)^{-1} \prod_{r=9}^{\infty}\left(1-q_{r}^{-3}\right)^{-1} \text {, }
$$

if $17 \nmid n, 19 \nmid n$.

Since $1<\left(1-19^{-3}\right)^{-1}<\left(1-17^{-3}\right)^{-1}$, it follows that in all the three cases, we have

$$
\begin{aligned}
\prod_{r=0}^{k}\left(1-p_{r}^{-\left(\alpha_{r}+1\right)}\right)^{-1}< & \left(1-5^{-2}\right)^{-1}\left(1-3^{-3}\right)^{-1}\left(1-13^{-3}\right)^{-1}\left(1-17^{-3}\right)^{-1} \\
& \quad \times \prod_{r=9}^{\infty}\left(1-q_{r}^{-3}\right)^{-1} \\
= & \frac{318897}{354046} \cdot \zeta(3) .
\end{aligned}
$$

If $\alpha_{1} \geqq 6$, then

$$
\begin{aligned}
\prod_{r=0}^{k}\left(1-p_{r}^{-\left(\alpha_{r}+1\right)}\right)^{-1} & <\left(1-5^{-2}\right)^{-1}\left(1-3^{-7}\right)^{-1} \prod_{r=6}^{\infty}\left(1-q_{r}^{-8}\right)^{-1} \\
& <\frac{318897}{354046} \cdot \zeta(3) .
\end{aligned}
$$

Hence, by $(A),(\boldsymbol{\gamma})$ follows in the case $\left(b_{1.1 .2}\right)$ also.

$\left(b_{1.8}\right)$ Suppose $p_{0} \neq 5$. Then $p_{2}=5$. In this case, as in $\left(a_{2}\right), p_{0} \neq 13$. For, otherwise, it would follow that $7 \mid n$, which can not hold. Since $p_{0}$ is of the form $4 \lambda+1, p_{0} \geqq 17$. $p_{r} \geqq q_{r+2}$ for $3 \leqq r \leqq k$. Hence 


$$
\begin{aligned}
\prod_{r=0}^{k}\left(1-p_{r}^{-\left(\alpha_{r}+1\right)}\right)^{-1} & <\left(1-17^{-2}\right)^{-1}\left(1-3^{-3}\right)^{-1}\left(1-5^{-3}\right)^{-1} \prod_{r=5}^{\infty}\left(1-q_{r}^{-3}\right)^{-1} \\
& <\frac{318897}{354046} \cdot \zeta(3) .
\end{aligned}
$$

Hence, by $(\mathrm{A}),(\gamma)$ follows in this case also. Thus $(\gamma)$ is proved.

$\left(b_{2}\right)$ Suppose $5 \nmid n$. Since $p_{0}$ is of the form $4 \lambda+1, p_{0} \geqq 13$. Also, $p_{0} \neq 29$. For, otherwise, it would follow as in $\left(a_{2}\right)$ that $\left(1+p_{0}\right) / 2$ $=3 \cdot 5 \mid n$, and this implies that $5 \mid n$, which is not the case.

$\left(b_{2.1}\right)$ If $7 \mid n$, then $p_{2}=7$.

$\left(b_{2.1 .1}\right)$ Suppose $\alpha_{1}=\alpha_{2}=2$. Then both 13 and 19 divide $n$, since $\sigma\left(p_{1}^{\alpha_{1}}\right)=13, \sigma\left(p_{2}^{\alpha_{2}}\right)=57=3.19$ and $\sigma(n)=2 n$. In this case, neither 11 nor 17 divides $n$. For, otherwise, it would follow that

$$
\sum_{p ! n} \frac{1}{p}>\frac{1}{3}+\frac{1}{7}+\frac{1}{13}+\frac{1}{17}+\frac{1}{19}>\frac{1}{3}+\frac{1}{338}+\log \left(\frac{18}{13}\right) .
$$

a contradiction to (iv). Hence, either (1) $p_{0}=13, p_{3}=19, p_{r} \geqq q_{r+5}$ for $4 \leqq r \leqq k$; or (2) $p_{0} \neq 13, p_{3}=13, p_{4}=19, p_{r} \geqq q_{r+4}$ for $5 \leqq r \leqq k$. In the second case, $p_{0} \geqq 37$, since $p_{n}$ is of the form $4 \lambda+1, p_{0} \neq 13,17 \nmid n$ and $p_{0} \neq 29$. In the first case,

$$
\begin{aligned}
\prod_{r=0}^{k}\left(1-p_{r}^{-\left(\alpha_{r}+1\right)}\right)^{-1}< & \left(1-13^{-2}\right)^{-1}\left(1-3^{-3}\right)^{-1}\left(1-7^{-3}\right)^{-1}\left(1-19^{-3}\right)^{-1} \\
& \times \prod_{r=9}^{\infty}\left(1-q_{r}^{-3}\right)^{-1} \\
= & \frac{1853074104}{2125240975} \cdot \zeta(3) .
\end{aligned}
$$

In the second case,

$$
\begin{aligned}
\prod_{r=0}^{k}\left(1-p_{r}^{-\left(\alpha_{r}+1\right)}\right)^{-1}< & \left(1-37^{-2}\right)^{-1}\left(1-3^{-3}\right)^{-1}\left(1-7^{-3}\right)^{-1}\left(1-13^{-3}\right)^{-1} \\
& \times\left(1-19^{-3}\right)^{-1} \prod_{r=9}^{\infty}\left(1-q_{r}^{-3}\right)^{-1} \\
< & \frac{1853074104}{2125240975} \cdot \zeta(3) .
\end{aligned}
$$

$\left(b_{2.1 .2}\right)$ If at least one of $\alpha_{1}$ and $\alpha_{2}$ is not equal to 2, then either $\alpha_{1} \geqq 4, \alpha_{2} \geqq 4$ or $\alpha_{1} \geqq 4, \alpha_{2}=2$ or $\alpha_{1}=2, \alpha_{2} \geqq 4$. The proofs for the first two cases are omitted as they are similar to the previous proofs. In both these cases, we easily verify that the upper bound obtained for 
$\bigcup_{p / n} p /(p-1)$ is less than the bound obtained in the third case. In the third case, we have

$$
\begin{aligned}
\prod_{r=0}^{k}\left(1-p_{r}^{-\left(\alpha_{r}+1\right)}\right)^{-1} & \\
& <\left(1-13^{-2}\right)^{-1}\left(1-3^{-3}\right)^{-1}\left(1-7^{-5}\right)^{-1} \prod_{r=5}^{\infty}\left(1-q_{r}^{-3}\right)^{-1} \\
& <\frac{1853074104}{2125240975} \cdot \zeta(3) .
\end{aligned}
$$

Hence, by $(A),(\delta)$ follows in any case under $\left(b_{2.1}\right)$.

$\left(\mathrm{b}_{2.2}\right)$ If $7 \nmid n$, then $p_{r} \geqq q_{r+3}$ for $2 \leqq r \leqq k$. Hence

$$
\begin{aligned}
\prod_{r=0}^{k}\left(1-p_{r}^{-\left(\alpha_{r}+1\right)}\right)^{-1} & <\left(1-3^{-3}\right)^{-1} \prod_{r=5}^{\infty}\left(1-q_{r}^{-3}\right)^{-1} \\
& <\frac{1853074104}{2125240975} \cdot \zeta(3) .
\end{aligned}
$$

Hence, by (A), $(\delta)$ follows in this case also. Thus $(\delta)$ is proved. Thus the proof of the theorem is complete.

\section{REFERENCES}

1. H. J. Kanold, Folgerungen aus dem Vorkommen einer Gauss'schen Primzahl in der Primfaktorenzerlegung einer ungeraden vollkommenen Zahl, J. Reine Angew. Math. 186 (1944), 25-29.

2. U. Kühnel, Verschärfung der notwendigen Bedingungen für die Existenz von ungeraden vollkommenen Zahlen, Math. Z. 52 (1949), 202-211.

3. P. J. McCarthy, Odd perfect numbers, Scripta Math. 23 (1957), 43-47.

4. D. Suryanarayana and N. Venkateswara Rao, On odd perfect numbers, Math. Student 29 (1961), 133-137.

5. D. Suryanarayana, On odd perfect numbers. II, Proc. Amer. Math. Soc. 14 (1963), 896-904.

6. J. Touchard, On prime numbers and perfect numbers, Scripta Math. 19 (1953), $35-39$.

ANDHRA UNIVERSITY, WALTAIR, INDIA 